\title{
Generating picosecond x-ray pulses in synchrotron light sources using dipole kickers
}

\author{
W. Guo* \\ NSLS II, Brookhaven National Laboratory, Upton, New York 11764, USA \\ B. Yang, C.-x. Wang, K. Harkay, and M. Borland \\ Argonne National Laboratory, 9700 South Cass Avenue, Argonne, Illinois 60439, USA \\ (Received 26 May 2006; published 1 February 2007)
}

\begin{abstract}
The duration of the x-ray pulse generated at a synchrotron light source is typically tens of picoseconds. Shorter pulses are highly desired by the users. In electron storage rings, the vertical beam size is usually orders of magnitude less than the bunch length due to radiation damping; therefore, a shorter pulse can be obtained by slitting the vertically tilted bunch. Zholents proposed tilting the bunch using rf deflection. We found that tilted bunches can also be generated by a dipole magnet kick. A vertical tilt is developed after the kick in the presence of nonzero chromaticity. The tilt was successfully observed and a 4.2-ps pulse was obtained from a 27-ps electron bunch at the Advanced Photon Source. Based on this principle, we propose a short-pulse generation scheme that produces picosecond x-ray pulses at a repetition rate of $1-2 \mathrm{kHz}$, which can be used for pump-probe experiments.
\end{abstract}

DOI: 10.1103/PhysRevSTAB.10.020701

PACS numbers: 41.85.Ct, 29.20.Dh, 29.27.Bd

\section{INTRODUCTION}

The x-ray pulse generated by synchrotron light sources has been widely used to explore material structure on the atomic scale. The duration of the pulse is on the order of $100 \mathrm{ps}$. Shorter pulses are highly desired for better resolution and fast dynamic process exploration. There are two thresholds for the snapshot of the dynamic process of atoms. One is the period of atom vibration $(\sim 100 \mathrm{fs})$; pulses of this duration can be used to follow the movement of atoms and molecules during chemical reaction and phase transition. Another threshold is for tracking electrons in atoms, which is of the order of attoseconds $\left(10^{-18} \mathrm{~s}\right)$.

Many efforts have been made to produce shorter x-ray pulses. Recently, subpicosecond sources of hard x-rays have been developed based on laser-produced plasma [1], higher harmonic generation [2], and relativistic Thomson scattering [3]. The photon flux generated by these methods is typically low. A much more promising source is a fourthgeneration light source, such as the Linac Coherent Light Source (LCLS) [4], which will produce 230-fs pulses at very high brightness.

As a third-generation light source, the Advanced Photon Source (APS) produces about 40-ps-long (rms) x-ray pulses. Besides high brightness, it also has the advantages of high reliability, high repetition rate, and energy tunability. It is of great interest to compress the pulse length by 2 to 3 orders of magnitude. An adjustable pulse length between 40 and 0.2 ps could be advantageous for some experiments even when the LCLS is available in 2008. Possible compression schemes for a synchrotron light

*Electronic address: wguo@bnl.gov source include femtosecond laser slicing [5], lower momentum compaction lattice [6], voltage or phase modulation [7], and rf deflection [8]. The first three methods either cannot offer sufficient compression or cannot maintain the current intensity or the photon flux. In principle, the $\mathrm{rf}$ deflection method can make full use of the flux. The idea is to apply a time-dependent vertical kick to the flatlying electron bunch using a high-frequency deflecting cavity. The generated $x$-ray beam therefore has verticallongitudinal $(y-z)$ correlation, or "tilt." The pulse can be compressed by arranging a $y$-dependent optical path with gratings or asymmetrically cut crystals. Therefore the flux can be preserved, and the final pulse length is determined by the bunch height, which is 3 orders of magnitude less than the bunch length.

The rf deflection method requires development of a new device. In this paper we show that a vertical tilt can also be created by a simple dipole magnet kick. In storage rings, the transverse and longitudinal motion is coupled due to chromaticity. We show that, if a bunch is kicked vertically, different longitudinal parts will have different betatron oscillation phases and a tilt can be developed. This is similar to the tilt observed in the head-tail instability [9] but in a controlled fashion. The tilt is fully developed in a half synchrotron period, and, in the case of linear motion, the vertical beam size along the tilt equals the original value. Therefore the effect is equivalent to rf deflection, except that it is not continuous. In reality, the beam size grows due to diffusion and nonlinearity, which limits the minimum achievable pulse length. Nonetheless, shorter pulses can still be obtained by either slitting or optical compression. In our experiment, we used a slit and observed a 7-times shorter pulse. In the following, we will 
discuss the synchrobetatron beam dynamics, present the experimental results, and propose a short-pulse generation scheme.

\section{SYNCHROBETATRON BEAM DYNAMICS AND DECOHERENCE}

In electron storage rings, if a particle receives a vertical kick $\Theta$ at $s_{0}$, the motion at location $s$ can be described by

$$
\begin{aligned}
y= & \exp \left(-a_{y} \theta\right) A(s) \sin \left[\int_{0}^{\theta} \nu_{y} d \theta+\psi_{s, 0}\right] \\
& +\sqrt{2 \beta_{y}(s) J_{y}} \cos \left[\int_{0}^{\theta} \nu_{y} d \theta+\psi_{s, 0}+\psi_{0}\right],
\end{aligned}
$$

where the first term is due to the kick and second term is the thermal motion. Here $\theta=2 n \pi(n=1,2,3, \ldots)$ is the orbital angle, $A(s)=\sqrt{\beta_{y}(s) \beta_{y}\left(s_{0}\right)} \Theta$ is the oscillation amplitude, $\beta_{y}$ is the beta function, $a_{y}$ is the vertical damping coefficient, $\psi_{s, 0}$ is the betatron phase advance from $s_{0}$ to $s$ in one turn, $\nu_{y}$ is the vertical tune, and $J_{y}$ and $\psi_{0}$ represent the initial action and angle due to the vertical emittance.

We note the amplitude and phase of the first term are the same for all the particles, and the phase dilutes with time due to the vertical tune spread. The vertical tune is related to the momentum and the oscillation amplitude due to nonlinearity. In first-order approximation,

$$
\nu_{y}=\nu_{y, 0}+C_{y} \delta+\mu J,
$$

where $\nu_{y, 0}$ is the constant tune, $\delta$ is the fractional momentum, $C_{y}=\frac{d \nu_{y}}{d \delta}$ is the vertical chromaticity, $\mu$ is the detuning factor, and $J$ is the action of the total betatron oscillation. $J$ is preserved in the case of linear betatron motion. From Eq. (1), $J=\frac{1}{2} P_{k}^{2}+J_{y}+P_{k} P_{y}$, where $P_{k}=\sqrt{\beta\left(s_{0}\right)} \Theta$ is the normalized kick amplitude, and $P_{y}=-\sqrt{2 J_{y}} \sin \psi_{0}$ is the initial normalized momentum. In this application the kick amplitude is much larger than the thermal motion amplitude, therefore $J \approx \frac{1}{2} P_{k}^{2}+P_{k} P_{y}$. The initial coordinate $P_{y}$ satisfies Gaussian distribution with $\sigma_{P_{y}}=\sqrt{\epsilon_{y}}$. Averaging Eq. (1) over $P_{y}$ and $\psi_{0}$ in the transverse phase space, one gets

$$
\begin{aligned}
\left\langle y\left(J_{y}, \psi_{0}\right)\right\rangle= & \exp \left(-a_{y} \theta-\frac{1}{2} a_{\mu} \theta^{2}\right) A(s) \\
& \times \sin \left(\nu_{y, 0} \theta+\frac{1}{2} \mu P_{k}^{2} \theta+\psi_{s, 0}+C_{y} \int \delta d \theta\right) .
\end{aligned}
$$

Equation (3) means the amplitude tune dependence introduces a $\theta^{2}$ exponential decay factor to the centroid oscillation and is characterized by the parameter $a_{\mu}=\mu^{2} P_{k}^{2} \epsilon_{y}$. The same result can be obtained if we simplify the formula in Ref. [10] using the condition $\mu \epsilon_{y} \theta \ll 1$. We believe the decoherence effect caused by vertical damping and excitation is small [11], therefore they are not included in the model.

Now let us examine the chromaticity term. The longitudinal motion is not affected by the transverse kick; therefore, $\delta$ undergoes harmonic oscillation and the particle distribution is in the equilibrium of damping and quantum excitation. We found that the betatron phase advance caused by chromaticity is well described by

$$
\begin{aligned}
\Delta \psi_{\delta}= & \int_{0}^{\theta} C_{y} \delta(\theta) d \theta \\
= & -\frac{C_{y}}{h \alpha_{c}}\left(1-e^{-a_{E} \theta} \cos \nu_{s} \theta\right) \Delta \phi(0) \\
& +\frac{C_{y}}{\nu_{s}} \delta(0) e^{-a_{E} \theta} \sin \nu_{s} \theta+\zeta_{n}(\theta),
\end{aligned}
$$

where $\Delta \phi$ is the relative rf phase, $a_{E}$ is the longitudinal damping coefficient, $\nu_{s}$ is the synchrotron tune, $h$ is the harmonic number, and $\alpha_{c}$ is the momentum compaction factor. The effect of damping and quantum excitation is twofold. First, it makes the integral independent of the initial state, which is described by the exponential decay factor. Second, it generates a random phase advance and is given by the $\zeta_{n}(\theta)$ term. $\zeta_{n}$ is a Gaussian-distributed noise; the subscript $n$ indicates that the value is different for different particles. $\zeta_{n}$ has the following properties:

$$
\left\langle\zeta_{n}\right\rangle=0, \quad \text { and } \quad\left\langle\zeta_{n}^{2}\right\rangle=\sigma_{\zeta}^{2}=\left(\frac{C_{y} \sigma_{\delta}}{\nu_{s}}\right)^{2}\left(1-e^{-2 a_{E} \theta}\right) \text {, }
$$

where the bracket \langle\rangle means average over all the particles, and $\sigma_{\delta}$ is the rms fractional momentum spread.

Assuming $\delta$ and $\Delta \phi$ all satisfy Gaussian distribution, and averaging Eq. (3) over $\delta$ and $\zeta$, one gets

$$
\langle y\rangle(\Delta \phi)=A(s) \exp \left(-\xi^{2}-a_{y} \theta\right) \sin \Upsilon,
$$

where

$$
\begin{gathered}
\xi^{2}=\frac{1}{2} a_{\mu} \theta^{2}+\frac{1}{2}\left(\frac{C_{y} \sigma_{\delta}}{\nu_{s}}\right)^{2}\left(1-e^{-2 a_{E} \theta} \cos ^{2} \nu_{s} \theta\right) \\
\Upsilon=\nu_{y, 0} \theta+\frac{1}{2} \mu P_{k}^{2} \theta+\psi_{s, 0}-\frac{C_{y}}{h \alpha_{c}}\left(1-e^{-a_{E} \theta} \cos \nu_{s} \theta\right) \Delta \phi .
\end{gathered}
$$

Therefore, the vertical displacement $\langle y\rangle(\Delta \phi)$ is related to the longitudinal position $\Delta \phi$, and a tilt can be formed. The betatron tune $\nu_{y, 0}$ and chromaticity $C_{y}$ can be adjusted such that the particles stay in the linear region of the sine function, hence the tilt is linear. 
Averaging further over $\Delta \phi$, one obtains

$$
\begin{aligned}
\langle y\rangle= & \exp \left[-a_{y} \theta-\frac{1}{2} a_{\mu} \theta^{2}-\left(\frac{C_{y} \sigma_{\delta}}{\nu_{s}}\right)^{2}\left(1-e^{-a_{E} \theta} \cos \nu_{s} \theta\right)\right] \\
& \times A(s) \sin \left(\nu_{y, 0} \theta+\frac{1}{2} \mu P_{k}^{2} \theta+\psi_{s, 0}\right) .
\end{aligned}
$$

Equation (9) means the kicked centroid oscillation decays due to radiation damping, amplitude tune dependence, and longitudinal diffusion. Take the APS as an example. The parameters are $a_{y}=6.1 \times 10^{-5}, a_{E}=1.2 \times 10^{-4}$, and $\epsilon_{y}=2.5 \times 10^{-11} \mathrm{~m}$. The detuning factor $\mu$ is typically $\sim 4 \times 10^{4} \mathrm{~m}^{-1}$ [12]. If $\beta_{y}\left(s_{0}\right)=20 \mathrm{~m}$ and the kick angle $\Theta=1 \times 10^{-4}$, one gets $a_{\mu}=8 \times 10^{-9}$. In several hundreds of turns $a_{\mu} \theta \ll a_{E}$, therefore the decoherence is dominated by the longitudinal diffusion in several synchrotron periods. This result is understandable. Because the vertical beam size is so small $(\sim 20 \mu \mathrm{m})$, all the particles have almost the same oscillation amplitude; therefore the amplitude tune spread is small.

One can also find the slice tilt angle

$$
\begin{aligned}
\theta_{y-z}(\Delta \phi) & =\frac{d\langle y\rangle(\Delta \phi)}{d \frac{c}{\omega} \Delta \phi} \\
& =\frac{\omega}{c} A(s) e^{-a_{y} \theta} \frac{C_{y}}{h \alpha_{c}}\left(1-\cos \nu_{s} \theta e^{-a_{E} \theta}\right) e^{-\xi^{2}} \cos \Upsilon,
\end{aligned}
$$

and the slice moment

$$
\begin{aligned}
\sigma_{y}^{2}(\Delta \phi)= & \beta_{y}(s) \epsilon_{y}+A^{2}(s) e^{-2 a_{y} \theta} \\
& \times \sinh \xi^{2} e^{-\xi^{2}}\left(1+e^{-2 \xi^{2}} \cos 2 \Upsilon\right),
\end{aligned}
$$

where $c$ is the speed of light and $\omega$ is the angular frequency of the rf system. From Eqs. (6) and (11), one gets the relation between $\sigma_{y}^{2}(\Delta \phi)$ and $\langle y\rangle(\Delta \phi)$ :

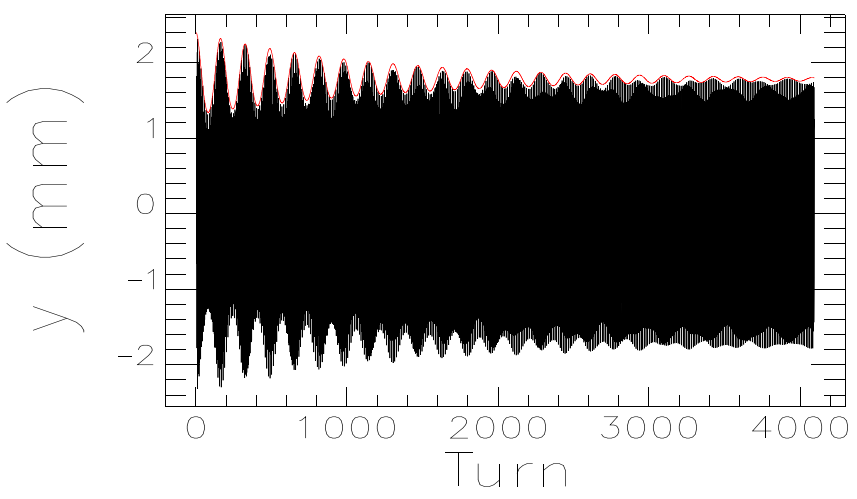

FIG. 1. (Color) Vertical decoherence due to longitudinal diffusion. The parameters are $C_{y}=3.5, \nu_{s}=0.0078, \sigma_{\delta}=9.6 \times$ $10^{-4}$, and $a_{E}=1.2 \times 10^{-4}$. The red envelop is given by Eq. (9) but with $\mu=0$ and $a_{y}=0$, the oscillation is obtained from simulation.

$$
\sigma_{y}^{2}(\Delta \phi)=\sigma_{y, 0}^{2}+\left(e^{-2 \xi^{2}}-1\right)\langle y\rangle^{2}(\Delta \phi),
$$

with $\sigma_{y, 0}^{2}=\beta_{y}(s) \epsilon_{y}+\frac{1}{2} \beta_{y}(s) \beta_{y}\left(s_{0}\right) \Theta^{2}\left(1-e^{-4 \xi^{2}}\right)$. Note this relation holds inside a bunch and is independent of the vertical tune.

Particle tracking was carried out to verify the above equations. Figure 1 shows the verification of the decoherence due to longitudinal diffusion. In the simulation, a linear one-turn map was used for vertical motion and vertical damping was not included. However, radiation damping and quantum excitation were included in the longitudinal mapping. The vertical tune was set to $\nu_{y}=$ $\nu_{y, 0}+C_{y} \delta$ to introduce synchrobetatron coupling. One can see from the plot that the analytical expression is in excellent agreement with the simulation. The other equations were also confirmed in the simulation; the results and the comparison with the beam position monitor signal will be shown in a separate paper in order not to divert from the topic.

\section{IMAGING THE TILTED BUNCH AND SLITTING FOR SHORTER PULSE}

In order to observe the tilted bunches, we conducted imaging experiments at the APS diagnostic beam line [13]. A dual-sweep streak camera was set up to take bunch profiles in the $(y, z)$ plane turn by turn. The pixel size is $y \times z=96 \times 364 \mu \mathrm{m}^{2}$, and the focus resolution is $\Delta y=$ $0.13 \mathrm{~mm}$ and $\Delta z=0.67 \mathrm{~mm}$. A vertical kicker kicks the beam at $2 \mathrm{~Hz}$ with a maximum strength of $0.15 \mathrm{mr}$. The vertical chromaticity was lowered from 6 to 3.5 to make the bunch tilt as linear as possible. The horizontal chromaticity was kept at 6.6 because it does not affect the bunch tilt. With the lowered chromaticity, we were able to store 1.3-mA current in a single bunch when the kicker was on.

A profile sweep was performed from turn 0 to 200 after the kick. The sine-shaped bunch was immediately observed. Figure 2 shows the side views of a bunch at several successive turns. Because the vertical tune $\nu_{y}=19.27$, the bunch flips in $y$ every other turn. One can see that the third bunch (counting from left) is in mirror symmetry to the first

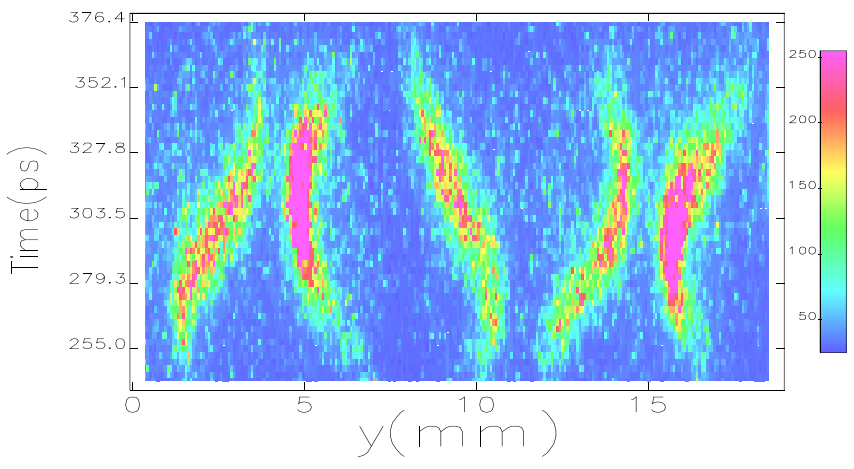

FIG. 2. (Color) The side views of a bunch at turn 63 to 67 after a vertical kick. 


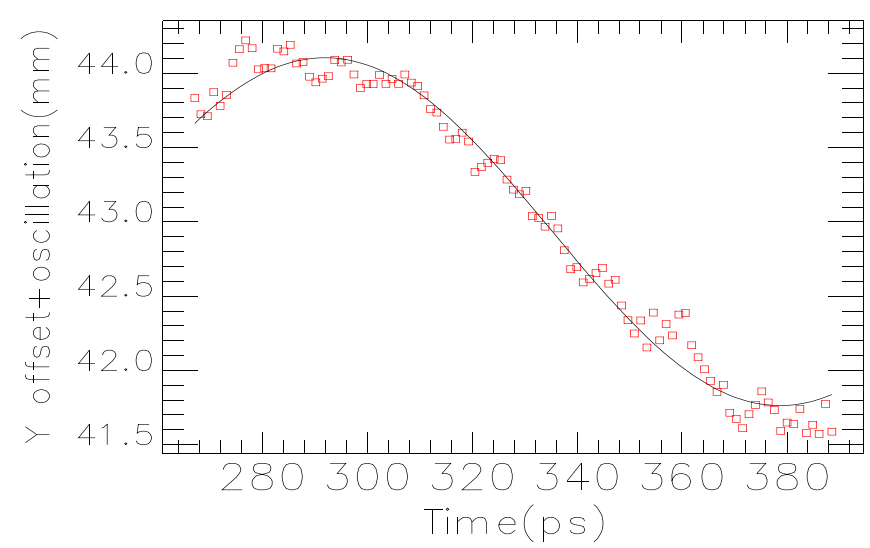

FIG. 3. (Color) The sinusoidal fit to the bunch slice centroid of turn 72. The fit function is $y=y_{0}+A_{y} \sin (\omega t+\phi), \omega=$ $23.35 \mathrm{ps}^{-1}$ is calculated from Eq. (6), $A_{y}=1.19 \mathrm{~mm}$ and $\phi=$ 2.92 are obtained from fitting. Equation (6) gives $A_{y, \text { cal }}=$ $1.15 \mathrm{~mm}$.

one, and the same symmetry occurs between the fourth and the second bunch. The slice width also varies with time, which makes the tilt disappear in a few tens of turns. The transverse profile of a slice is well fitted by a Gaussian function. From the fitting, the slice center $\langle y\rangle(\Delta \phi)$ and the slice rms width $\sigma_{y}(\Delta \phi)$ were obtained. Figure 3 shows the fitted and measured slice centroid of turn 72 . The theory gives excellent prediction on the amplitude and the oscillation frequency. Note the betatron phase cannot be determined because of a vertical tune jitter of $\sim 10^{-4}$ per turn. In the figure the betatron phase span is about $\pi$ from bunch head $\left(2 \sigma_{t}\right)$ to tail $\left(-2 \sigma_{t}\right)$ after half of a synchrotron period. According to Eq. (6), the phase span can be lowered by decreasing the chromaticity, which was also confirmed in the experiment.

Equation (12) states that there is a square relation between $\sigma_{y}^{2}$ and $\langle y\rangle$ inside a bunch. Figure 4 shows the fitting

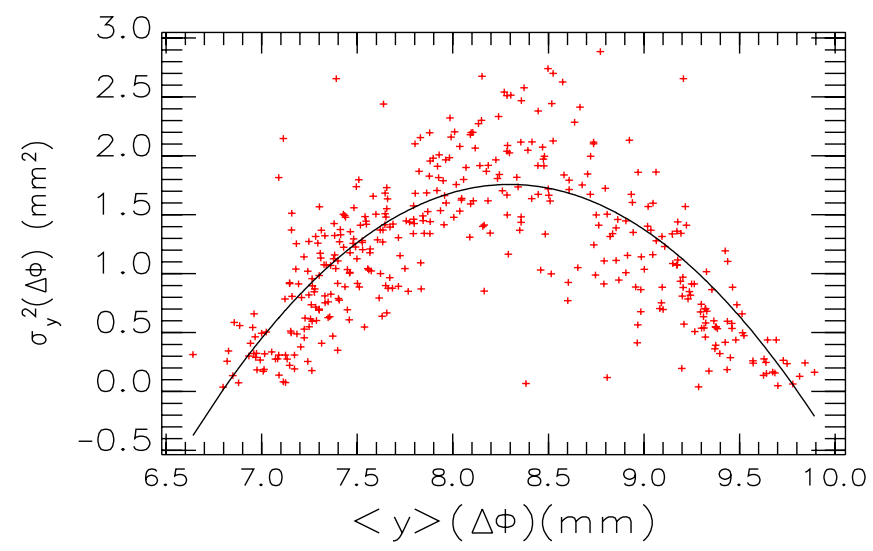

FIG. 4. (Color) The fit between $\sigma_{y}^{2}$ and $\langle y\rangle$ for turn 48. The red crosses are obtained from five images of the same turn, the black curve is a hypobola fit. The fit result is $\sigma_{y}^{2} \propto-0.775\langle y\rangle^{2}$. The predicted coefficient is -0.113 .

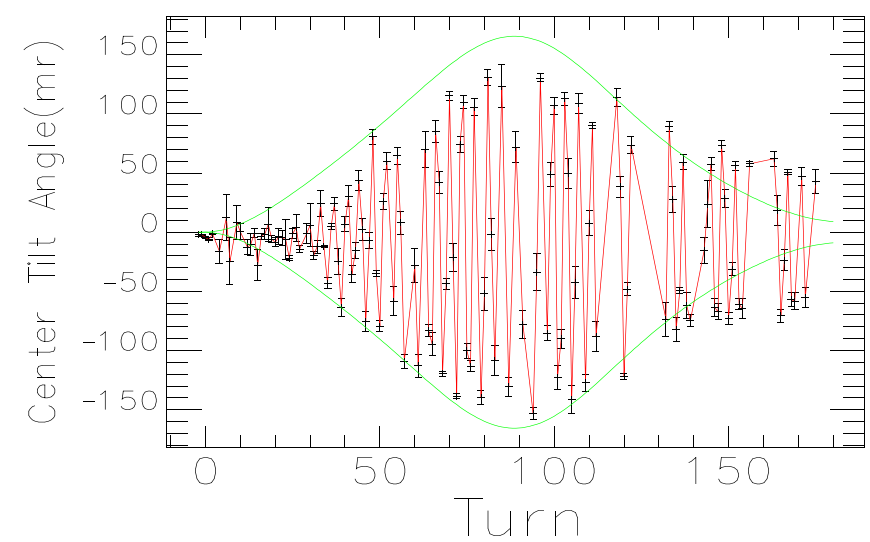

FIG. 5. (Color) The center angle changes with time.

between these two quantities for turn 48. There is a discrepancy between the fitting coefficient and the prediction. The reason is probably due to the streak camera resolution and various jitters. Note the crosses form a band in the plot, which is clearly an indication of these uncertainties.

The tilt angle of the bunch center is obtained from the sinusoidal fitting. The slice width and the tilt angle versus turn number are plotted in Figs. 5 and 6. The crosses with error bar are obtained from the images, and the envelop curves are calculated by Eqs. (10) and (11). We found that the modulation frequency is significantly smaller than the coherent synchrotron frequency, and it changes with rf voltage, chromaticity, and the stored current. We believe this is due to potential well distortion, indeed, at $1.3 \mathrm{~mA}$, the bunch length is significantly lengthened. The modulation tune goes to the synchrotron tune if the current is lowered. In the fitting, the modulation tune was adjusted, but nominal values were used for the other parameters. The agreement is still reasonable. There are discrepancies for the value of $\sigma_{y}^{2}$, and the possible reasons are: first, the measurement of the beam width is more susceptible to the streak camera resolution; second, there are other decoher-

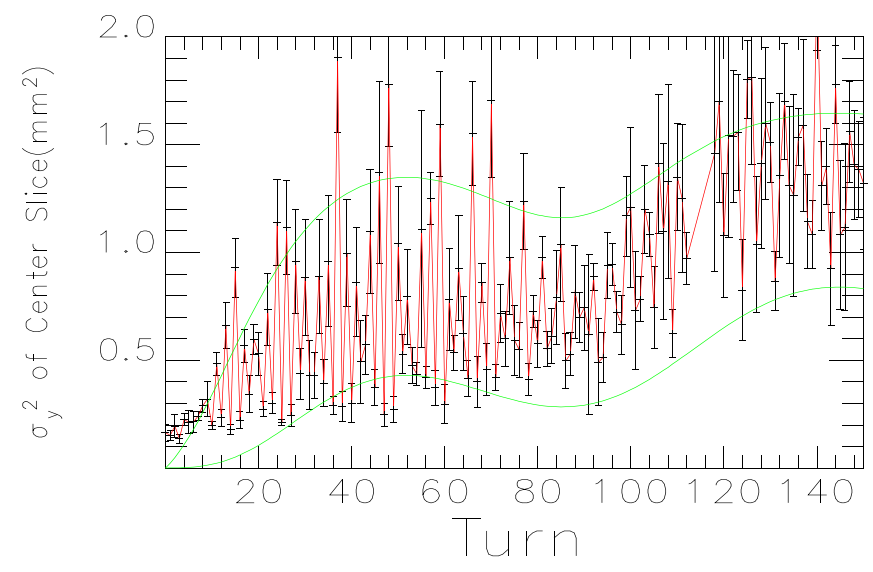

FIG. 6. (Color) The $\sigma_{y}^{2}$ of the center slice changes with turn number. 


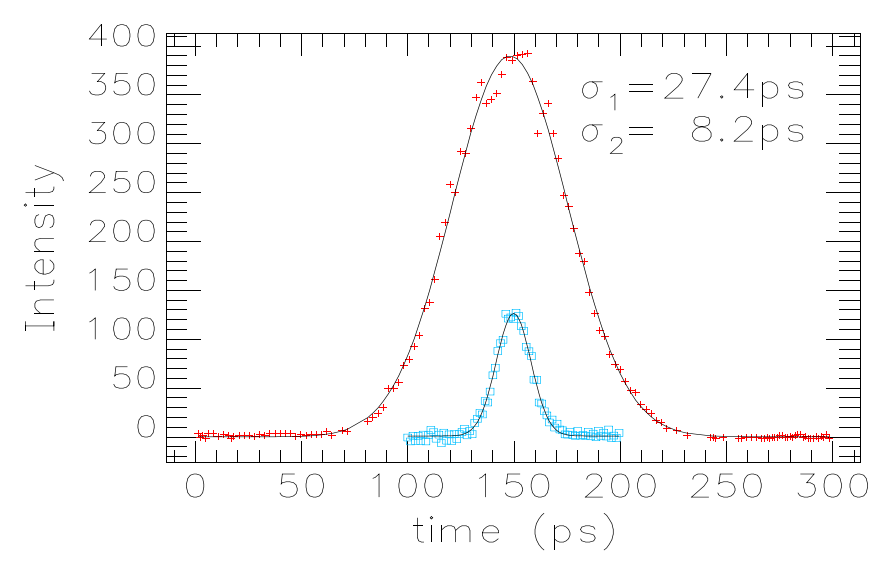

FIG. 7. (Color) The length of the long-time-overlapped pulses. Red crosses, without the slit; blue squares, with the slit.

ence mechanisms, such as wakefield, higher order chromaticity, and linear $x-y$ coupling that are not included in our model.

At half the modulation period, the center tilt reaches maximum and the slice emittance reaches a minimum; therefore, it is the best time to slice for short pulse. Note that, if there is no diffusion and nonlinearity, the bunch recoheres and the slice emittance would go back to the original value at that point. We changed the above experiment setup to observe the shortest possible pulse. A slit of $75 \pm 15-\mu \mathrm{m}$ effective width was used to clip the image right before the streak camera. Since the slit is very small compared to the oscillation amplitude, the tilt linearity was not a concern; therefore we set the vertical chromaticity to 4. A filter of $10 \times 550 \mathrm{~nm}$ was used to reduce the diffraction effect in the optics. Because the longitudinal scan frequency of the streak camera is one-third of the rf frequency, we injected ten $0.03-\mathrm{mA}$ bunches into every third bucket. The images from the ten bunches were overlapped to get a good profile fitting. At such a low current, we found the tilt reaches maximum at turn 65 , which is the nominal half synchrotron period.

Figure 7 shows the pulse length with and without the slit. The profiles are overlapped for 300 shots. The one without the slit gives the original bunch length, which is $27.4 \mathrm{ps}$. As a comparison, the zero current bunch length is about 20 ps. The one with the slit gives 8.2 ps. Even though each individual pulse is much shorter, the tune jitter changes the arrival time of the slitted pulse and results in a longer overlapped profile. One can calculate the corresponding tune jitter to be $\Delta \nu_{y}=1.6 \times 10^{-4}$, which agrees with the tune jitter measurement result. From the plot, one can calculate the slitted photon flux to be about $10 \%$ of the pulse. At very low current, the wakefield effect becomes negligible, and the decoherence is dominated by the longitudinal diffusion. In that case the pulse compression ratio is $R \approx \sqrt{2 /\left[1-\exp \left(-\pi a_{E} / \nu_{s}\right)\right]}$. Figure 8 shows the average slitted-pulse length when the time jitter is removed manually from the data. The value of $4.2 \mathrm{ps}$ is very close to

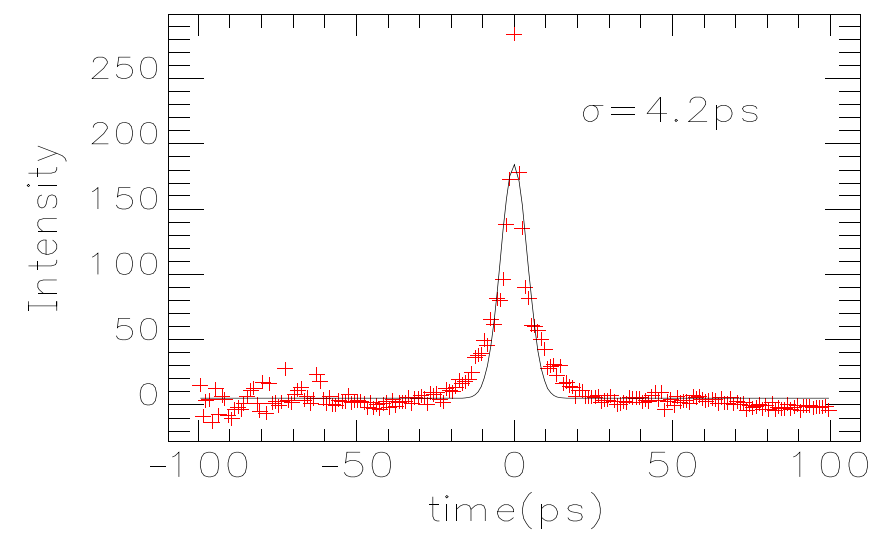

FIG. 8. (Color) The average length of the slitted pulse when the time jitter is removed.

the predicted value of $4 \mathrm{ps}$. This pulse length is of interest because the time jitter can be overcome with available techniques [14]. The time jitter can even be utilized to scan the interested interval.

\section{THE SHORT-PULSE GENERATION SCHEME}

Based on the above principle, we propose the short-pulse generation scheme illustrated in Fig. 9. $N$ bunches are stored in the ring, a fast kicker kicks one bunch (the black one) at a time, and a second kicker gives a reverse kick after one synchrotron period. A timing source controls the kick and sends a delayed signal to the detector. The gate of the detector opens only at the time when the tilt reaches maximum. The tilted pulse (the black dotted lines) goes through the focusing mirrors and the wavelength-filtering monochromator like the normal pulse (the blue long dashed lines). Because of the tilt, the shorter pulse can be obtained by an offset slit; therefore shorter pulse experiments can be performed in parallel. It takes a few damping time periods for the beam size to damp down. Other bunches can be kicked during the interval; therefore, the repetition rate will be $\sim N / \tau_{y}$. In such a setup, the vertical chromaticity does not have to be low as long as the kick amplitude is much bigger compared to the slit width. An additional advantage is that there is no need to change the X-ray optics.

Take the APS as an example. The tune jitter gives a 13\% residue of centroid oscillation after the second kick. More reverse kicks controlled by a real-time feedback can be applied to eliminate the residue. Because of the decoherence, the beam size increases by $40 \%$ of the kick amplitude. ELEGANT [15] simulation shows that a repetition rate of $1.2 \mathrm{kHz}$ can be realized with 24 bunches if the kick amplitude is less than $0.05 \mathrm{mr}$. The $1-2-\mathrm{kHz}$ frequency matches the laser frequency in pump-probe experiments. In order for the fast kickers to kick a single bunch, the pulse duration should be less than $0.3 \mu \mathrm{s}$. The flux of each pulse will be about $10 \%$ of a single bunch if an effective slit of 


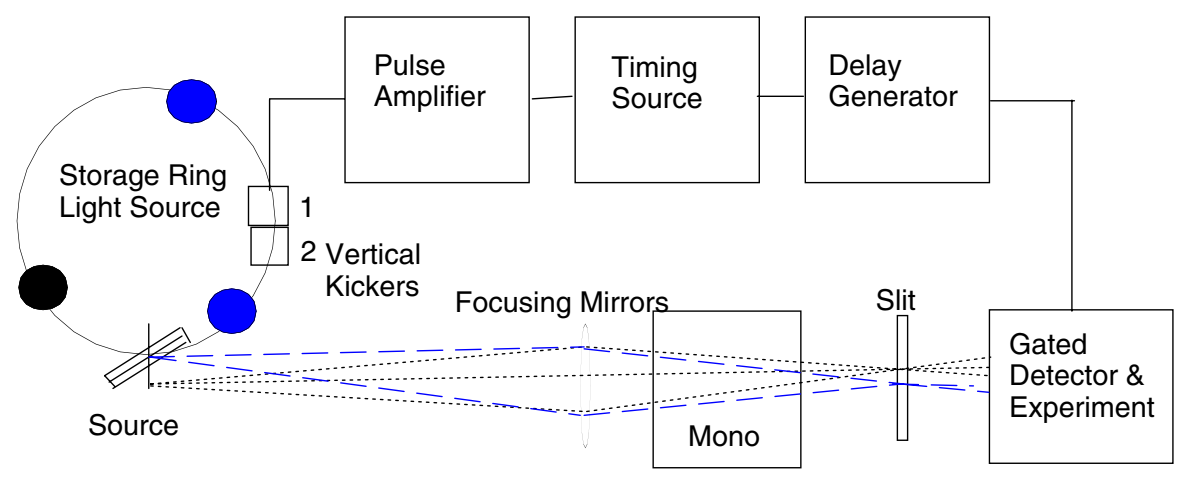

FIG. 9. (Color) The short-pulse generation scheme.

$50 \mu \mathrm{m}$ is used. The minimum pulse length will be about $3-$ $4 \mathrm{ps}$ for a bunch with 1-2-mA current. At higher current the pulse length is longer and the repetition rate has to be lower due to the wakefield decoherence and bunch lengthening.

There are methods to increase the photon flux. One way is to store 24 bunch trains instead of singlets. This requires the kicker pulse to be flattop; and the x-ray pulses might be lengthened due to the long-range wakefield. A second method is to replace the slit with a compression optics similar to that proposed in Ref. [8]. We note the tune jitter results in about $6 \%$ tilt angle change, therefore degrades the compression.

\section{SUMMARY}

In summary, we have found a novel mechanism to generate highly desired short x-ray pulses in a synchrotron radiation facility and demonstrated it experimentally at the APS. Based on the principle, we propose a scheme to produce picosecond $\mathrm{x}$-ray pulses at a repetition rate of 1$2 \mathrm{kHz}$ using two pulsed-magnet kickers. This simple and effective technique is applicable to any synchrotron light source.

\section{ACKNOWLEDGMENTS}

The authors would like to thank Vadim Sajaev for assistance with machine setups, and John Byrd, Samuel Krinsky, and Eric Landahl for helpful discussions. This work was supported by the U.S. Department of Energy, Office of Basic Energy Sciences, under Contracts No. W31-109-ENG-38 and No. DE-AC02-98CH10886.
[1] M. M. Murnane et al., Science 251, 531 (1991).

[2] M. Drescher et al., Nature (London) 419, 803 (2002).

[3] R. W. Schoenlein et al., Science 274, 236 (1996).

[4] J. Arthur et al., LINAC Coherent Light Source (LCLS) Design Study Report, SLAC-R-0521, 1998.

[5] A. Zholents and M. Zolotorev, Phys. Rev. Lett. 76, 912 (1996); R. W. Schoenlein et al., Science 287, 2237 (2000).

[6] J. Feikes, K. Holldack, P. Kuske and G. Wustefeld, Proceedings of EPAC 2004, p. 1954; Y. Shoji, S. Hisao, and T. Matsubara, Proceedings of EPAC 2004, p. 2356; J. Feikes, K. Holldack, P. Kuske, and G. Wustefeld, Beam Dynamics Newsletter 35, 82 (2004).

[7] D. Li et al., Phys. Rev. E 48, R1638 (1993); M. Bai et al., Phys. Rev. ST Accel. Beams 3, 064001 (2000); G. Decker et al., Phys. Rev. ST Accel. Beams 9, 120702 (2006).

[8] A. Zholents et al., Nucl. Instrum. Methods Phys. Res., Sect. A 425, 385 (1999).

[9] J. Gareyte and F. Sacherer, Proceedings of the 9th International Conference on High Energy Accelerators, Stanford, 1974, p. 341.

[10] R.E. Miller et al., SSC Report No. SSC-N-360, 1987; M. G. Minty, A.W. Chao, and W.L. Spence, in Proceedings of the 1995 Particle Accelerator Conference, 1995, p. 3037.

[11] H. Moshammer, Phys. Rev. E 48, 1390 (1993); 48, 2140 (1993).

[12] C.-x. Wang, in Proceedings of the 2003 Particle Accelerator Conference, 2003, p. 3407.

[13] B. Yang and A. Lumpkin, Rev. Sci. Instrum. 67, 3367 (1996).

[14] A. L. Cavalieri et al., Phys. Rev. Lett. 94, 114801 (2005).

[15] M. Borland, Advanced Photon Source LS-287, 2000. 